\title{
Aborder les vecteurs sociaux et structurels du VIH au Canada
}

\author{
Rourke $\mathrm{SB}^{1,2,3^{*}}$, Bacon $\mathrm{J}^{1}$, McGee $\mathrm{F}^{4}$, Gilbert $\mathrm{M}^{1,5}$ \\ ${ }^{1}$ Réseau ontarien de traitement du VIH, Université de Toronto, Toronto (Ontario) \\ ${ }^{2}$ Département de psychiatrie, Université de Toronto, Toronto (Ontario) \\ ${ }^{3}$ Centre for Research on Inner City Health, Li Ka Shing Knowledge Institute de I'hôpital St. Michael's, Toronto (Ontario) \\ ${ }^{4}$ Bureau de lutte contre le sida du ministère ontarien de la Santé et des Soins de longue durée, Toronto (Ontario) \\ ${ }^{5}$ Dalla Lana School of Public Health, Université de Toronto, Toronto (Ontario) \\ ${ }^{*}$ Correspondance: sean.rourke@utoronto.ca
}

\section{Résumé}

Il existe de nouveaux espoirs que nous pouvons considérablement réduire les taux du virus de l'immunodéficience humaine $(\mathrm{VIH})$. L'organisation de lutte contre le syndrome d'immunodéficience acquise (sida) des Nations Unies, le Programme commun des Nations Unies sur le VIH/SIDA, a encouragé tous les pays à s'efforcer d'atteindre des objectifs audacieux qui pourraient considérablement infléchir la courbe relative aux infections à VIH et aux décès causés par le $\mathrm{VIH}: 90 \%$ des personnes vivant avec le VIH qui ont reçu un diagnostic; $90 \%$ des personnes ayant reçu un diagnostic sous traitement; et $90 \%$ des personnes sous traitement et dont la charge virale est indétectable. Ce nouvel optimisme repose en grande partie sur les excellents résultats des recherches selon lesquels un traitement précoce et continu du VIH améliore les résultats sur la santé individuelle et réduit la charge virale des personnes, ce qui les rend moins infectieuses. Cependant, le risque d'infection au VIH est loin d'être réparti uniformément entre les populations les plus vulnérables. Ces populations à risque auront le plus de difficulté à atteindre ces objectifs, car elles sont prises dans une syndémie (épidémie synergique) consistant à entrelacer les problèmes de santé et les problèmes sociaux. Nos recherches, et celles des autres, démontrent que les personnes qui sont dans une syndémie de problèmes de santé mentale, de dépendance et de problèmes sociaux concomitants ( $p$. ex. sans-abrisme, insécurité alimentaire) sont beaucoup plus susceptibles d'arrêter le traitement, sont moins susceptibles de respecter le traitement et sont moins susceptibles d'atteindre ou de maintenir une charge virale indétectable. Des études d'intervention ont démontré qu'une approche combinée en matière de prévention et de traitement du VIH qui va au-delà des soins primaires et des outils de santé mentale inclut des interventions sociales et structurelles qui ont un effet protecteur et qu'elle peut réduire le risque et améliorer l'observance. Les personnes vivant avec le VIH et à risque de le contracter ont besoin d'un meilleur accès aux services sociaux, services de santé mentale et services de traitement clinique qui les aideront à atteindre et maintenir un niveau optimal de santé et de bien-être. Nous encourageons fortement les gens œuvrant dans le secteur du VIH à l'échelle du pays à définir une vision commune avec des buts et objectifs clairs. Grâce à des efforts concertés et ciblés, un accent sur le programme et la science de la mise en œuvre, et une volonté de voir et traiter le VIH comme un problème social et biomédical, la quatrième décennie du VIH au Canada pourrait bien être la dernière.

\section{L'occasion}

À ce chapitre, pour ce qui est de la quatrième décennie du VIH, il y a le renouvellement de l'espoir et de l'énergie. Le Programme commun des Nations Unies sur le VIH/SIDA (ONUSIDA) a encouragé tous les pays à s'efforcer d'atteindre des objectifs audacieux qui pourraient considérablement infléchir la courbe relative aux infections au $\mathrm{VIH}$ et aux décès causés par le $\mathrm{VIH}: 90 \%$ des personnes vivant avec le $\mathrm{VIH}$ qui ont reçu un diagnostic; $90 \%$ des personnes ayant reçu un diagnostic sous traitement; et $90 \%$ des personnes sous traitement et dont la charge virale est indétectable (1). Plusieurs pays, comme les 
États-Unis et l'Australie, États individuels et provinces (p. ex. la Colombie-Britannique) et villes (p. ex. San Francisco) ont lancé des stratégies ambitieuses axées sur le traitement afin de réduire et même mettre fin aux nouvelles infections au VIH.

Cet optimisme repose en grande partie sur les excellents résultats des recherches selon lesquels un traitement précoce et continu du VIH améliore les résultats sur la santé individuelle et réduit la charge virale des personnes, ce qui les rend moins infectieuses. Des études récentes ont également révélé la capacité de la prophylaxie préexposition pour prévenir les infections chez les personnes qui courent un risque élevé de contracter le VIH $(2,3)$.

L'amélioration efficace du dépistage et du traitement du VIH est essentielle. Cependant, comme la Commission ONUSIDA-Lancet a récemment indiqué, il «... ne suffira pas à contrôler l'épidémie » si nous n'abordons pas le stigmate, la discrimination, les syndémies et les vecteurs sociaux et structurels de l'épidémie du VIH (4).

\section{L'incidence des syndémies}

Le risque d'infection au VIH est loin d'être réparti uniformément entre les populations les plus vulnérables. II est concentré chez les personnes prises dans une syndémie (épidémie synergique) consistant à entrelacer les problèmes de santé et les problèmes sociaux. Par exemple, dans l'étude de cohorte multicentrique sur le sida de 4295 hommes gais, bisexuels et autres hommes ayant des relations sexuelles avec d'autres hommes (HARSAH), Mimiaga et al. se sont penchés sur les effets de cinq conditions psychosociales sur l'incidence du VIH : des symptômes dépressifs, une consommation excessive d'alcool, l'utilisation de stimulants, la consommation de drogues multiples et la violence sexuelle pendant l'enfance (5). Comparativement aux personnes n'ayant aucun de ces problèmes de santé psychosociaux, un nombre croissant de ces conditions a été fortement lié à l'incidence du VIH. Les hommes ayant quatre ou cinq de ces conditions présentaient 8,7 fois plus de risque de contracter le $\mathrm{VIH}$ sur une période de 48 mois. Des recherches canadiennes semblables vont plus loin en décrivant comment les syndémies sociales des expériences de marginalisation (p. ex. harcèlement, discrimination de carrière, violence physique, rapports sexuels forcés, tendances suicidaires) contribuent de façon fondamentale à la prévalence de conditions psychosociales semblables parmi les HARSAH (6).

Dans le cadre de notre étude de cohorte du Réseau ontarien de traitement du VIH (I'OHTN) qui suit plus de 5000 personnes en Ontario (environ un tiers de toutes les personnes recevant actuellement des soins), nous sommes encouragés de constater que les personnes qui font partie de cette cohorte de soins et qui reçoivent des soins dans les cliniques de soins spécialisés contre le VIH se rapprochent des objectifs 90-90-90 pour avoir suivi un traitement antirétroviral et pour avoir une charge virale indétectable (7). Cependant, une image différente et inquiétante se dessine lorsqu'on examine les personnes recevant des soins présentant plusieurs risques syndémiques psychosociaux. À l'échelle de toutes les populations, les personnes qui ont connu une syndémie de problèmes de santé mentale et de dépendance concomitants sont beaucoup plus susceptibles d'arrêter le traitement, sont moins susceptibles de respecter le traitement et sont moins susceptibles d'atteindre ou de maintenir une charge virale indétectable (8). (Remarque : Les personnes vivant avec le $\mathrm{VIH}$ qui ne font pas partie de cette cohorte pourraient ne pas se porter aussi bien en ce qui concerne les objectifs 90-90-90. L'Ontario analyse actuellement la cascade des deux tiers restants des personnes vivant avec le $\mathrm{VIH}$ qui reçoivent des soins.) Les autres provinces ont également constaté que ces facteurs complexes ont une incidence sur les résultats en matière de santé. Par exemple, le Southern Alberta HIV Program a constaté une prévalence élevée de violence familiale et conjugale à l'échelle de tous les groupes à risque d'infection par le $\mathrm{VIH}$, y compris les HARSAH, et a consigné ses effets négatifs sur la participation aux soins liés au VIH, la santé mentale et la stabilité sociale $(9,10)$.

Nous avons également pris conscience de l'incidence des déterminants sociaux sur la santé mentale et les résultats liés au VIH. D'après nos recherches sur le logement en Ontario, lesquelles étaient financées par les Instituts de recherche en santé du Canada (IRSC) et l'OHTN, les personnes vivant avec le VIH qui ont un logement instable sont plus susceptibles de ce qui suit : avoir des charges virales plus élevées; ne pas respecter le traitement du VIH; ne pas accéder aux services médicaux ou sociaux; avoir des problèmes de 
consommation d'alcool et de drogues; présenter des niveaux de dépression et de stress plus élevés; et avoir des taux de mortalité plus élevés. En fait, la dépression liée au VIH est $50 \%$ plus élevée chez les personnes qui avaient de la difficulté à prendre en charge les frais liés au logement, et $60 \%$ plus élevée chez les personnes qui estimaient qu'elles n'appartenaient pas à leur quartier. De plus, les personnes qui se préoccupaient d'être obligées de déménager étaient significativement plus susceptibles de développer une dépression récurrente $(8,11)$. Ces résultats soulignent que les réseaux et contextes sociaux sont importants : Iorsqu'ils ne sont pas sécuritaires, encourageants et favorables, ils peuvent exposer les personnes au risque d'autres conséquences négatives sur la santé.

Les recherches menées aux États-Unis et au Canada ont démontré des incidences semblables liées à l'insécurité alimentaire (12-14). Les personnes vivant avec le VIH qui souffrent d'insécurité alimentaire sont moins susceptibles d'avoir des charges virales indétectables, ont des taux de morbidité et de mortalité plus élevés et ont une qualité de vie liée à la santé physique et mentale inférieure (15). Elles manquent davantage de rendez-vous de soins primaires et ont moins tendance à recourir aux traitements du VIH. Toutefois, une fois qu'elles reçoivent des services d'alimentation et de nutrition efficaces, la situation change radicalement : elles sont moins susceptibles de manquer des rendez-vous, sont plus susceptibles de poursuivre leur traitement, obtiennent un meilleur score en ce qui concerne la santé mentale et sont plus susceptibles d'avoir une charge virale indétectable.

\section{Utilisation de la science des programmes pour aborder les vecteurs sociaux et structurels}

L'incidence des syndémies renforce l'importance d'une approche combinée à la prévention et au traitement du VIH qui va au-delà des soins primaires et des outils de la santé mentale (16) pour inclure des interventions sociales et structurelles protectrices qui peuvent réduire le risque et améliorer l'observance - par exemple les programmes Logement d'abord, l'accès aux programmes de réduction des méfaits, les interventions pour réduire la violence, et des environnements sociaux favorables qui aident à éliminer la stigmatisation et la discrimination et à protéger les droits de la personne. La science des programmes et la science de la mise en œuvre offrent de nouvelles façons de penser aux moyens pragmatiques de mobiliser les résultats de recherche et de créer des interventions efficaces, adaptables et viables dans le monde réel (17-21). La science des programmes est l'« application systématique des connaissances scientifiques théoriques et empiriques pour améliorer la conception, la mise en œuvre et l'évaluation des programmes de santé publique » (19). La science de la mise en œuvre est une "spécialité multidisciplinaire qui recherche des connaissances généralisées sur le comportement des intervenants, des organisme, des collectivités et des individus afin de comprendre l'ampleur, les raisons et les stratégies pour combler l'écart entre les preuves et les pratiques de base pour la santé dans des contextes réels » (17).

II ne fait aucun doute que le Canada possède les connaissances, les outils et les ressources pour fournir ce type d'approche programmatique et combinée qui peut contribuer à enrayer l'épidémie du VIH dans le pays. En Ontario, notre nouvelle stratégie sur le VIH souligne l'importance de travailler en collaboration avec tous les groupes d'intervenants (communauté, santé publique et secteurs de la santé) pour offrir aux personnes vivant avec le $\mathrm{VIH}$ et à risque de le contracter un meilleur accès aux services sociaux, aux services de santé mentale et aux services de traitement clinique qui les aideront à atteindre et maintenir un niveau optimal de santé et de bien-être.

\section{Période des approches combinées à l'échelle du système}

Nous encourageons fortement les gens œuvrant dans le secteur du VIH à l'échelle du pays à collaborer pour apprendre les uns des autres et pour définir une vision commune ayant des buts et objectifs clairs et les structures et les interventions qui contribueront à établir une approche intersectorielle et combinée, tout en mettant l'accent sur la science des programmes et de la mise en œuvre.

Sur le plan clinique, cela signifie que, en plus d'offrir des services de dépistage et de traitement, le médecin de premier recours ou le médecin de famille ou de salle d'urgence posera systématiquement des questions 
aux patients vivant avec le $\mathrm{VIH}$ ou à risque de le contracter à propos de leur logement, sécurité alimentaire, expérience de violence, santé mentale et consommation d'alcool et de drogues, puis suggérera des aiguillages appropriés. Au niveau des programmes, cela signifie l'introduction d'interventions sociales et structurelles efficaces ainsi que l'évaluation de leurs répercussions dans différents contextes et populations. Au niveau des politiques, cela signifie l'élaboration de politiques, comme des indicateurs pour les résultats sociaux, qui permettront au système de soins de santé de mettre en œuvre des interventions combinées efficaces.

\section{Message à retenir}

Les approches biomédicales ne suffiront pas à elles seules à mettre fin au $\mathrm{VIH}$. Nous devons aborder les vecteurs sociaux et structurels. Grâce à des efforts concertés et ciblés, un accent sur la science des programmes et la mise en œuvre de la science, et une volonté de voir et traiter le VIH comme un problème social et biomédical, la quatrième décennie du VIH au Canada pourrait bien être la dernière.

\section{Remerciements}

Les auteurs tiennent à remercier toutes les personnes vivant avec le VIH qui ont offert de participer à l'étude de cohorte de l'OHTN le travail et le soutien des membres passés et présents du comité de gouvernance de l'étude, le personnel infirmier et les médecins qui ont appuyé la collecte des données. Nous tenons en outre à remercier les laboratoires de santé publique Ontario pour le maintien des liens avec la base de données des tests de charge virale du $\mathrm{VIH}$.

\section{Conflit d'intérêts}

\section{Aucun}

\section{Financement}

L'étude de cohorte de l'OHTN est financée par le Bureau de lutte contre le sida du ministère de la Santé et des Soins de longue durée de l'Ontario. Le financement des études de recherche en santé concernant le logement et la sécurité alimentaire a été assuré par les Instituts de recherche en santé du Canada (IRSC). Les constatations, les opinions et les conclusions exprimées sont celles des auteurs et n'engagent en aucune manière les IRSC ni le ministère de la Santé et des Soins de longue durée de l'Ontario.

\section{Références}

(1) Joint United Nations Programme on AIDS (UNAIDS). 90-90-90-An ambitious treatment target to help end the AIDS epidemic. Geneva: UNAIDS; 2014 [cited 2015 Oct 20]. http://www.unaids.org/sites/default/files/media_asset/90-90-90_en_0.pdf

(2) Grant RM, Lama JR, Anderson PL, McMahan V, Liu AY, Vargas L, et al. Preexposure chemoprophylaxis for HIV prevention in men who have sex with men. N Engl J Med. 2010;363(27):2587-99.

(3) Baeten JM, Donnell D, Ndase P, Mugo NR, Campbell JD, Wangisi J, et al. Antiretroviral prophylaxis for HIV prevention in heterosexual men and women. N Engl J Med. 2012;367(5):399-410.

(4) Piot P, Abdool Karim SS, Hecht R, Legido-Quigley H, Buse K, Stover J, et al. A UNAIDS-Lancet Commission on Defeating AIDS—Advancing global health. Lancet. 2015 Jul 11;386(9989):171-218.

(5) Mimiaga MJ, O'Cleirigh C, Biello KB, Robertson AM, Safren SA, Coates TJ, et al. The effect of psychosocial syndemic production on 4-year HIV incidence and risk behavior in a large cohort of sexually active men who have sex with men. J Acquir Immune Defic Syndr. 2015 Mar 1;68(3):329-36.

(6) Ferlatte O, Hottes TS, Trussler T, Marchand R. Evidence of a syndemic among young Canadian gay and bisexual men: Uncovering the associations between anti-gay experiences, psychosocial issues, and HIV risk. AIDS Behav. 2014 Jul;18(7):1256-63.

(7) Burchell AN, Gardner S, Light L, Ellis BM, Antoniou T, Bacon J, et al. Implementation and operational research: Engagement in HIV care among persons enrolled in a clinical HIV cohort in Ontario, Canada, 2001-2011. J Acquir Immune Defic Syndr. 2015 Sep 1;70(1):e10-9.

(8) Rourke SB. Social and structural drivers affecting the health of people living with HIV. The North American Housing and HIVIAIDS Research Summit. Summit VIII: Tackling the Social Drivers of HIV; 2015 Sep 14-16; Washington, DC. 
(9) Siemieniuk RA, Miller P, Woodman K, Ko K, Krentz H, Gill MJ. Prevalence, clinical associations, and impact of intimate partner violence among HIV-infected gay and bisexual men: A population-based study. HIV Med. 2013 May;14(5):293-302.

(10) Siemieniuk RA, Krentz HB, Miller P, Woodman K, Ko K, Gill MJ. The clinical implications of high rates of intimate partner violence against HIV-positive women. J Acquir Immune Defic Syndr. 2013 Sep 1;64(1):32-8. Choi S. Impacts of housing-related conditions on prevalence, recurrence, and incidence of current depression among people living with HIV in Ontario over a five-year follow-up: Results from the Ontario HIV Treatment Network Cohort Study. The North American Housing and HIVIAIDS Research Summit. Summit VIII: Tackling the Social Drivers of HIV; 2015 Sep 14-16; Washington, DC.

(12) Anema A, Vogenthaler N, Frongillo EA, Kadiyala S, Weiser SD. Food insecurity and HIVIAIDS: Current knowledge, gaps, and research priorities. Curr HIVIAIDS Rep. 2009 Nov;6(4):224-31.

(13) Weiser SD, Fernandes KA, Brandson EK, Lima VD, Anema A, Bangsberg DR, et al. The association between food insecurity and mortality among HIV-infected individuals on HAART. J Acquir Immune Defic Syndr. 2009 Nov 1;52(3):342-9.

(14) Anema A, Chan K, Chen Y, Weiser S, Montaner JS, Hogg RS. Relationship between food insecurity and mortality among HIV-positive injection drug users receiving antiretroviral therapy in British Columbia, Canada. PLoS ONE. 2013;8(5):e61277.

(15) Choi SK, Fielden S, Globerman J, Koornstra JJ, Hambly K, Walker G, et al. Food insufficiency, housing and health-related quality of life: Results from the Positive Spaces, Healthy Places study. AIDS Care. 2015 Sep;27(9):1183-90.

(16) Beyrer C, Sullivan PS, Sanchez J, Dowdy D, Altman D, Trapence G, et al. A call to action for comprehensive HIV services for men who have sex with men. Lancet. 2012 Jul 28;380(9839):424-38.

(17) Odeny TA, Padian N, Doherty MC, Baral S, Beyrer C, Ford N, et al. Definitions of implementation science in HIVIAIDS. Lancet HIV. 2015 May;2(5):e178-80.

(18) Glasgow RE, Chambers D. Developing robust, sustainable, implementation systems using rigorous, rapid and relevant science. Clin TransI Sci. 2012 Feb;5(1):48-55.

(19) Blanchard JF, Aral SO. Program science: An initiative to improve the planning, implementation and evaluation of HIV/sexually transmitted infection prevention programmes. Sex Transm Infect. 2011 Feb;87(1):2-3.

(20) Aral SO, Blanchard JF. The program science initiative: Improving the planning, implementation and evaluation of HIV/STI prevention programs. Sex Transm Infect. 2012 Apr;88(3):157-9.

(21) Glasgow RE, Vinson C, Chambers D, Khoury MJ, Kaplan RM, Hunter C. National Institutes of Health approaches to dissemination and implementation science: Current and future directions. Am J Public Health. 2012 Jul;102(7):1274-81. 